\title{
This article has been retracted:
} Radiotherapy Technique can be Important on Survival in Patients with Gastric Cancer Treated with Postoperative Chemoradiotherapy

\section{(D) Beyza Şirin ÖZDEMiR}

Department of Radiation Oncology, Akdeniz university, Antalya-Turkey

The Turkish Society for Radiation Oncology regrets to inform that the above mentioned article (Radiotherapy Technique can be Important on Survival in Patients with Gastric Cancer Treated with Postoperative Chemoradiotherapy) has been retracted based on author request as her declared that the data provided in the article and copied from another study by mistake. This error was recognized only after the publication. 